\title{
Front Matter: Volume 7258
}

, "Front Matter: Volume 7258," Proc. SPIE 7258, Medical Imaging 2009:

Physics of Medical Imaging, 725801 (26 March 2009); doi: 10.1117/12.827591

SPIE Event: SPIE Medical Imaging, 2009, Lake Buena Vista (Orlando Area), Florida, United States 


\title{
PROGRESS IN BIOMEDICAL OPTICS AND IMAGING
}

Vol. 10 , No. 34

\section{Medical Imaging 2009 \\ Physics of Medical Imaging}

\author{
Ehsan Samei \\ Jiang Hsieh \\ Editors
}

9-12 February 2009

Lake Buena Vista, Florida, United States

Sponsored by

SPIE

Cooperating Organizations

AAPM-American Association of Physicists in Medicine (United States)

APS-American Physiological Society (United States)

CARS-Computer Assisted Radiology and Surgery (Germany)

IS\&T-The Society for Imaging Science and Technology

MIPS-Medical Image Perception Society (United States)

RSNA-Radiological Society of North America (United States)

SIIM-Society for Imaging Informatics in Medicine (United States)

SMl-The Society for Molecular Imaging

The DICOM Standards Committee (United States)

Published by

SPIE

Part One of Three Parts

Volume 7258

Proceedings of SPIE, 1605-7422, v. 7258

SPIE is an international society advancing an interdisciplinary approach to the science and application of light. 
The papers included in this volume were part of the technical conference cited on the cover and title page. Papers were selected and subject to review by the editors and conference program committee. Some conference presentations may not be available for publication. The papers published in these proceedings reflect the work and thoughts of the authors and are published herein as submitted. The publisher is not responsible for the validity of the information or for any outcomes resulting from reliance thereon.

Please use the following format to cite material from this book:

Author(s), "Title of Paper," in Medical Imaging 2009: Physics of Medical Imaging, edited by Ehsan Samei, Jiang Hsieh, Proceedings of SPIE Vol. 7258 (SPIE, Bellingham, WA, 2009) Article CID Number.

ISSN 1605-7422

ISBN 9780819475091

Published by

SPIE

P.O. Box 10, Bellingham, Washington 98227-0010 USA

Telephone +1 3606763290 (Pacific Time) · Fax +1 3606471445

SPIE.org

Copyright @ 2009, Society of Photo-Optical Instrumentation Engineers.

Copying of material in this book for internal or personal use, or for the internal or personal use of specific clients, beyond the fair use provisions granted by the U.S. Copyright Law is authorized by SPIE subject to payment of copying fees. The Transactional Reporting Service base fee for this volume is $\$ 18.00$ per article (or portion thereof), which should be paid directly to the Copyright Clearance Center (CCC), 222 Rosewood Drive, Danvers, MA 01923. Payment may also be made electronically through CCC Online at copyright.com. Other copying for republication, resale, advertising or promotion, or any form of systematic or multiple reproduction of any material in this book is prohibited except with permission in writing from the publisher. The CCC fee code is 1605$7422 / 09 / \$ 18.00$.

Printed in the United States of America.

Publication of record for individual papers is online in the SPIE Digital Library.

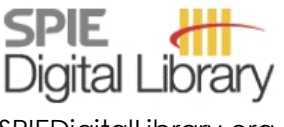

SPIEDigitalLibrary.org

Paper Numbering: Proceedings of SPIE follow an e-First publication model, with papers published first online and then in print and on CD-ROM. Papers are published as they are submitted and meet publication criteria. A unique, consistent, permanent citation identifier (CID) number is assigned to each article at the time of the first publication. Utilization of CIDs allows articles to be fully citable as soon they are published online, and connects the same identifier to all online, print, and electronic versions of the publication. SPIE uses a six-digit CID article numbering system in which:

- The first four digits correspond to the SPIE volume number.

- The last two digits indicate publication order within the volume using a Base 36 numbering system employing both numerals and letters. These two-number sets start with 00, 01, 02, 03, 04, $05,06,07,08,09,0 \mathrm{~A}, 0 \mathrm{~B} \ldots \mathrm{OZ}$, followed by 10-1Z, 20-2Z, etc.

The CID number appears on each page of the manuscript. The complete citation is used on the first page, and an abbreviated version on subsequent pages. Numbers in the index correspond to the last two digits of the six-digit CID number. 


\section{Contents}

\section{Part One}

xxv Conference Committee

xxvii In Memoriam: Robert F. Wagner (1938-2008)

SESSION 1 KEYNOTE AND CT DOSE: TRIBUTE TO BRUCE HASEGAWA

725802 Pediatric CT and radiation: our responsibility (Keynote Paper) [7258-01]

D. P. Frush, Duke Univ. Medical Ctr. (United States)

725803 Low radiation dose C-arm cone-beam CT based on prior image constrained compressed sensing (PICCS): including compensation for image volume mismatch between multiple data acquisitions [7258-02]

B. Nett, J. Tang, B. Aagaard-Kienitz, H. Rowley, G.-H. Chen, Univ. of Wisconsin, Madison (United States)

$725804 \quad$ Patient-specific dose estimation for pediatric abdomen-pelvis CT [7258-03]

X. Li, E. Samei, W. P. Segars, G. M. Sturgeon, Duke Univ. (United States); J. G. Colsher, GE Healthcare (United States); D. P. Frush, Duke Univ. (United States)

\section{SESSION 2 CT PERFORMANCE}

725805 Analytical construction of 3D NPS for a cone beam CT system [7258-04] J. Baek, N. J. Pelc, Stanford Univ. (United States)

725806 Evaluation of the additive noise of a flat panel detector and its effect on cone-beam CT applications [7258-05]

K. Yang, S.-Y. C. Huang, N. J. Packard, J. M. Boone, Univ. of California, Davis (United States)

$725807 \quad$ Noise analysis in intensity-weighted region-of-interest imaging for cone-beam CT [7258-06] S. Cho, E. Pearson, C. A. Pelizzari, X. Pan, The Univ. of Chicago (United States)

725808 Optimization of 4D cone-beam CT: evaluation of streaking artifacts and noise with various simulated gantry rotation speeds [7258-07]

M. Ahmad, P. Balter, The Univ. of Texas M.D. Anderson Cancer Ctr. (United States); P. Munro, Varian Medical Systems, Inc. (United States); J. Lu, Virginia Commonwealth Univ. (United States); T. Pan, The Univ. of Texas M.D. Anderson Cancer Ctr. (United States)

725809 Measurement of spatial and density resolutions in x-ray nanocomputed tomography [7258-08]

Y. Kawata, K. Kageyama, Univ. of Tokushima (Japan); Y. Nakaya, Shizuoka Cancer Ctr. (Japan); N. Niki, Univ. of Tokushima (Japan); K. Umetani, Japan Synchrotron Radiation Research Institute (Japan); K. Yada, Tohken Co. Ltd. (Japan); H. Ohmatsu, National Cancer Ctr. Hospital East (Japan); K. Eguchi, Teikyo Univ. (Japan); M. Kaneko, National Cancer Ctr. Hospital (Japan); N. Moriyama, National Cancer Ctr. Research Ctr. for Cancer Prevention and Screening (Japan) 
7258 OA Simulation and analysis of image quality impact from single-source ultra-wide coverage CT scanner [7258-09]

B. Li, T. L. Toth, J. Hsieh, X. Tang, P. Crandall, R. F. Senzig, General Electric Healthcare (United States)

\section{SESSION 3 CT APPLICATIONS}

7258 OB Four-dimensional cardiac reconstruction from rotational $x$-ray sequences: first results for 4D coronary angiography [7258-10]

E. Hansis, Philips Research Europe (Germany) and Univ. of Karlsruhe (Germany);

H. Schomberg, K. Erhard, Philips Research Europe (Germany); O. Dössel, Univ. of Karlsruhe (Germany); M. Grass, Philips Research Europe (Germany)

7258 OC High temporal resolution cardiac cone-beam CT using a slowly rotating C-arm gantry [7258-11]

G.-H. Chen, J. Tang, B. Nett, S. Leng, J. Zambelli, Z. Qi, N. Bevins, S. Reeder, H. Rowley, Univ. of Wisconsin, Madison (United States)

7258 OD Flash imaging in dual source CT (DSCT) [7258-12]

H. Bruder, M. Petersilka, H. Mehldau, W. Heidinger, T. Allmendinger, B. Schmidt, R. Raupach,

C. Thierfelder, K. Stierstorfer, T. Flohr, Siemens Healthcare (Germany)

7258 OE Kyphoplasty interventions using a navigation system and C-arm CT data: first clinical results [7258-13]

M. Hoheisel, Siemens AG Healthcare (Germany); M. Skalej, O. Beuing, Univ. Hospital Magdeburg (Germany); U. Bill, K. Klingenbeck-Regn, Siemens AG Healthcare (Germany); R. Petzold, M. H. Nagel, CAS innovations GmbH \& Co. KG (Germany)

7258 OF Cone-beam CT using a C-arm system as front end and a spherical spiral as source trajectory [7258-14]

H. Schomberg, Philips Research Europe (Germany); P. van de Haar, W. Baaten, Philips Healthcare (Netherlands)

7258 OG Quantization of liver tissue in dual kVp computed tomography using linear discriminant analysis [7258-15]

J. E. Tkaczyk, D. Langan, X. Wu, D. Xu, T. Benson, J. D. Pack, A. Schmitz, GE Global Research (United States); A. Hara, W. Palicek, Mayo Clinic Scottsdale (United States); P. Licato, J. Leverentz, GE Healthcare (United States)

$7258 \mathrm{OH} \quad$ Patient specific computerized phantoms to estimate dose in pediatric CT [7258-16] W. P. Segars, Duke Univ. (United States); G. Sturgeon, Duke Univ. (United States) and Univ. of North Carolina at Chapel Hill (United States); X. Li, Duke Univ. (United States); L. Cheng, C. Ceritoglu, J. T. Ratnanather, M. I. Miller, B. M. W. Tsui, The Johns Hopkins Univ. (United States); D. Frush, E. Samei, Duke Univ. (United States) 
7258 Ol Three-dimensional imaging properties of rotation-free square and hexagonal micro-CT systems [7258-17]

E. Quan, D. S. Lalush, North Carolina State Univ. (United States) and The Univ. of North Carolina at Chapel Hill (United States)

7258 0J Enhanced computer simulation of CT mammography using a flat-panel imager [7258-18] C. S. Didier, J. M. O'Connor, Univ. of Massachusetts Medical School (United States) and Univ. of Massachussetts at Lowell (United States); Y. Chen, Walter Reed Army Medical Ctr. (United States); S. J. Glick, Univ. of Massachusetts Medical School (United States)

7258 OK Volume and tissue composition preserving deformation of breast CT images to simulate breast compression in mammographic imaging [7258-19]

T. Han, L. Chen, C. Lai, X. Liu, Y. Shen, Y. Zhong, S. Ge, Y. Yi, T. Wang, C. C. Shaw, The Univ. of Texas M.D. Anderson Cancer Ctr. (United States)

7258 OL Breast density measurement: 3D cone beam computed tomography (CBCT) images versus 2D digital mammograms [7258-20]

T. Han, C.-J. Lai, L. Chen, X. Liu, Y. Shen, Y. Zhong, S. Ge, Y. Yi, T. Wang, W. T. Yang, C. C. Shaw, The Univ. of Texas M.D. Anderson Cancer Ctr. (United States)

7258 OM Visibility of microcalcifications in CCD-based cone beam CT: a preliminary study [7258-21] Y. Shen, L. Chen, S. Ge, Y. Yi, T. Han, Y. Zhong, C.-J. Lai, X. Liu, T. Wang, C. C. Shaw, The Univ. of Texas M.D. Anderson Cancer Ctr. (United States)

\section{SESSION $5 \quad$ BREAST TOMOSYNTHESIS}

725800 Estimating breast tomosynthesis performance in detection tasks with variable-background phantoms [7258-23]

S. Young, College of Optical Sciences, The Univ. of Arizona (United States); S. Park, S. K. Anderson, A. Badano, K. J. Myers, CDRH, U.S. Food and Drug Administration (United States); P. Bakic, Univ. of Pennsylvania (United States)

7258 OP Optimized lesion detection in digital breast tomosynthesis [7258-24]

A. S. Chawla, E. Samei, J. Y. Lo, Duke Univ. (United States)

$72580 Q \quad$ Nonuniform angular dose distribution in digital breast tomosynthesis for increased conspicuity of small high contrast objects [7258-25]

Y.-H. Hu, W. Zhao, Stony Brook Univ. (United States)

7258 OR The effect of lag on image quality for a digital breast tomosynthesis system [7258-26]

J. G. Mainprize, X. Wang, Sunnybrook Health Sciences Ctr. (Canada); M. J. Yaffe,

Sunnybrook Health Sciences Ctr. (Canada) and Univ. of Toronto (Canada)

7258 OS Computerized 3D breast phantom with enhanced high-resolution detail [7258-27]

C. M. Li, W. P. Segars, J. Y. Lo, Duke Univ. (United States) and Duke Univ. Medical Ctr. (United States); A. I. Veress, Univ. of Washington (United States); J. M. Boone, Univ. of California, Davis (United States); J. T. Dobbins III, Duke Univ. (United States) and Duke Univ. Medical Ctr.

(United States) 
7258 OT Blind motion compensation for positron-emission-tomography [7258-28]

M. Blume, Instituto de Física Corpuscular (Spain) and Technische Univ. München (Germany); A. Keil, N. Navab, Technische Univ. München (Germany); M. Rafecas, Instituto de Física Corpuscular (Spain)

7258 OU Joint image reconstruction and nonrigid motion estimation with a simple penalty that encourages local invertibility [7258-29]

S. Y. Chun, J. A. Fessler, Univ. of Michigan (United States)

7258 OV Direct reconstruction of PET receptor binding parametric images using a simplified reference tissue model [7258-30]

G. Wang, J. Qi, Univ. of California, Davis (United States)

7258 OW Advanced reconstruction of attenuation maps using SPECT emission data only [7258-31] A. Salomon, Philips Research Labs. (Germany) and RWTH Aachen Univ. (Germany);

A. Goedicke, Philips Research Labs. (Germany); T. Aach, RWTH Aachen Univ. (Germany)

7258 OX HyperSPECT: a new system for pre-clinical imaging in vivo [7258-32]

S. Tibbelin, P. Nillius, B. Cederström, M. Danielsson, The Royal Institute of Technology

(Sweden)

\section{SESSION 7 NON-X-RAY IMAGING}

7258 OY Novel insight into magnetic resonance through a spherical coordinate framework for the Bloch equation [7258-33]

B. Tahayori, L. A. Johnston, I. M. Y. Mareels, P. M. Farrell, The Univ. of Melbourne (Australia)

$7258 \mathrm{OZ}$ Regularization of parallel MRI reconstruction using in vivo coil sensitivities [7258-34] Q. Duan, R. Otazo, New York Univ. School of Medicine (United States); J. Xu, Siemens Medical Solutions USA Inc. (United States); D. K. Sodickson, New York Univ. School of Medicine (United States)

725810 Quantification of three-dimensional tongue motion during speech using zHARP [7258-35] X. Liu, Johns Hopkins Univ. (United States); J. Zhuo, Univ. of Maryland School of Medicine (United States); H. Agarwal, K. Z. Abd-Elmoniem, Johns Hopkins Univ. (United States); E. Murano, M. Stone, Univ. of Maryland School of Medicine (United States); R. Gullapalli, Univ. of Maryland School of Dentistry (United States); J. L. Prince, Johns Hopkins Univ. (United States)

725811 Detection of iron overload through neutron stimulated emission computed tomography: a sensitivity analysis study [7258-36]

A. J. Kapadia, Duke Univ. Medical Ctr. (United States)G. A. Agasthya, G. D. Tourassi, Duke Univ. Medical Ctr. (United States) and Duke Univ. (United States)

725812 Iterative finite-element-based inversion for quantified detection of molecular targets using optoacoustic tomography [7258-37]

T. Jetzfellner, D. Razansky, A. Rosenthal, R. Schulz, K.-H. Englmeier, V. Ntziachristos, Institute for Biological and Medical Imaging (Germany) and Helmholtz Ctr. Munich (Germany) 
725813 Simulating ultra-wideband imaging for the early detection of tissue injury [7258-38] W. Niu, Oakland Univ. (United States); K. Havaty, Central Michigan Univ. (United States); D. Tanska, N. Tseng, Univ. of Michigan (United States); J. Li, B. Penprase, Oakland Univ. (United States)

\section{SESSION $8 \quad X$-RAY DETECTORS}

725814 Active pixel and photon counting imagers based on poly-Si TFTs: rewriting the rule book on large area flat panel $x$-ray devices [7258-39]

L. E. Antonuk, M. Koniczek, Y. El-Mohri, Q. Zhao, Univ. of Michigan (United States)

725815 Characterization of current programmed amorphous silicon active pixel sensor readout circuit for dual mode diagnostic digital x-ray imaging [7258-134]

N. Safavian, M. Yazdandoost, D. Wu, A. Sultana, M. H. Izadi, K. S. Karim, A. Nathan, Univ. of Waterloo (Canada); J. A. Rowlands, Univ. of Waterloo (Canada) and Thunder Bay Regional Research Institute Lakehead Univ. (Canada)

725816 Amorphous selenium detector utilizing a Frisch grid for photon-counting imaging applications [7258-41]

A. H. Goldan, Y. Fang, K. S. Karim, Univ. of Waterloo (Canada); O. Tousignant, H. Mani,

L. Laperrière, Anrad Corp. (Canada)

725817 Component analysis of a new solid state x-ray image intensifier (SSXII) using photon transfer and instrumentation noise equivalent exposure (INEE) measurements [7258-42]

A. Kuhls-Gilcrist, D. R. Bednarek, S. Rudin, Toshiba Stroke Research Ctr., Univ. at Buffalo (United States)

725818 Quantitative radiography enabled by slot collimation and a novel scatter correction technique on a large-area flat panel $\mathbf{x}$-ray detector [7258-43]

M. L. Yue, A. E. Boden, J. M. Sabol, GE Healthcare (United States)

\section{SESSION 9 RADIOGRAPHY AND MAMMOGRAPHY PERFORMANCE}

725819 Noise and signal detection in digital x-ray detectors using the spatial definition of SNR [7258-44]

I. S. Kyprianou, A. Badano, S. Park, H. Liu, K. J. Myers, CDRH, U.S. Food and Drug

Administration (United States)

7258 1A Extension of DQE to include scatter, grid, magnification, and focal spot blur: a new experimental technique and metric [7258-45]

N. T. Ranger, Duke Univ. Medical Ctr. (United States); A. Mackenzie, King's College Hospital (United Kingdom); I. D. Honey, Guy's and St. Thomas' Hospital (United Kingdom);

J. T. Dobbins III, C. E. Ravin, E. Samei, Duke Univ. Medical Ctr. (United States)

7258 1B Noise analysis of full field digital mammography systems [7258-46]

V. Ravaglia, Ctr. per lo Studio e la Prevenzione Oncologica (Italy); R. Bouwman, Radboud Univ. Medical Ctr. (Netherlands); K. Young, The Royal Surrey County Hospital (United Kingdom); R. van Engen, Radboud Univ. Medical Ctr. (Netherlands); B. Lazzari, Ctr. per lo Studio e la Prevenzione Oncologica (Italy) 
7258 1C Predicting contrast detail performance from objective measurements in digital mammography [7258-47]

K. C. Young, The Royal Surrey County Hospital (United Kingdom) and Univ. of Surrey (United Kingdom); A. Alsager, Univ. of Surrey (United Kingdom); D. R. Dance, J. M. Oduko,

O. Gundogdu, The Royal Surrey County Hospital (United Kingdom); N. M. Spyrou, Univ. of Surrey (United Kingdom)

7258 1D SKE/BKE task-based methodology for calculating Hotelling observer SNR in mammography [7258-48]

H. Liu, Univ. of Maryland, College Park (United States) and CDRH, U.S. Food and Drug Administration (United States); I. S. Kyprianou, A. Badano, K. J. Myers, R. J. Jennings, S. Park, R. V. Kaczmarek, K. Chakrabarti, CDRH, U.S. Food and Drug Administration (United States)

SESSION 10 PHOTON-COUNTING AND DIRECT-CONVERSION SYSTEMS

7258 IG Feasibility of amorphous selenium based photon counting detectors for digital breast tomosynthesis [7258-51]

J. Chen, J. Lehnert, Stoney Brook Univ. (United States); P. O'Connor, G. De Geronimo, Brookhaven National Lab. (United States); E. Dolazza, Analogic Corp. (United States); O. Tousignant, L. Laperrière, J. Greenspan, Anrad Corp. (Canada); W. Zhao, Stoney Brook Univ. (United States)

$7258 \mathrm{IH}$ Evaluation of the $\mathrm{x}$-ray response of amorphous selenium coated 100 - $\mu \mathrm{m}$ pitch a-Si active pixel sensors for tomosynthesis applications [7258-52]

F. Taghibakhsh, Sunnybrook Health Sciences Ctr. (Canada) and Univ. of Toronto (Canada); D. M. Hunter, Sunnybrook Health Sciences Ctr. (Canada); K. S. Karim, Univ. of Waterloo (Canada); G. Belev, S. O. Kasap, Univ. of Saskatchewan (Canada); V. Verpakhovski, Sunnybrook Health Sciences Ctr. (Canada); M. J. Yaffe, Sunnybrook Health Sciences Ctr. (Canada) and Univ. of Toronto (Canada)

725811 Direct-conversion $\mathbf{5 0} \boldsymbol{\mu m}$ pixel-pitch detector for digital mammography using amorphous selenium as a photoconductive switching layer for signal charge readout [7258-53] K. Irisawa, K. Yamane, S. Imai, M. Ogawa, T. Shouji, T. Agano, Y. Hosoi, T. Hayakawa, FUJIFILM Corp. (Japan)

7258 1J A photon-counting detector for dual-energy breast tomosynthesis [7258-54] E. Fredenberg, Royal Institute of Technology (Sweden); M. Lundqvist, M. Åslund, M. Hemmendorff, Sectra Mamea AB (Sweden); B. Cederström, M. Danielsson, Royal Institute of Technology (Sweden)

\section{SESSION 11 TOMOSYNTHESIS}

$7258 \mathrm{IK} \quad$ First physical measurements and clinical evaluation for long-view tomosynthesis [7258-55] D. Notohara, K. Nishino, K. Shibata, Shimadzu Corp. (Japan) 
7258 1L A task-based evaluation method for $x$-ray breast imaging systems using variablebackground phantoms [7258-56]

S. Park, CDRH, U.S. Food and Drug Administration (United States); H. Liu, Univ. of Maryland, College Park (United States); R. Jennings, CDRH, U.S. Food and Drug Administration (United States); R. Leimbach, Marquette Univ. (United States); I. Kyprianou, A. Badano, K. Myers, CDRH, U.S. Food and Drug Administration (United States)

$72581 \mathrm{M}$ Analysis of lung nodule detectability and anatomical clutter in tomosynthesis imaging of the chest [7258-57]

S. Yoon, Stanford Univ. (United States); J. G. Gang, Univ. of Toronto (Canada); D. J. Tward, Ontario Cancer Institute, Princess Margaret Hospital (Canada); J. H. Siewerdsen, Univ. of Toronto (Canada) and Ontario Cancer Institute, Princess Margaret Hospital (Canada);

R. Fahrig, Stanford Univ. (United States)

7258 iN Multiple information tomosynthesis with grating-based phase contrast imaging [7258-58] Z. Wang, Z. Huang, Y. Xiao, L. Zhang, K. Kang, Tsinghua Univ. (China)

725810 Imaging of small children with a prototype for photon counting tomosynthesis [7258-59] L. del Risco Norrlid, E. Fredenberg, Royal Institute of Technology (Sweden); M. Hemmendorff, Sectra Mamea AB (Sweden); C. Jackowski, Univ. Bern (Switzerland); M. Danielsson, Royal Institute of Technology (Sweden)

7258 1P The impact of anisotropic sampling on the MTF reconstruction in limited aperture tomosynthesis [7258-60]

H. Souchay, R. Klausz, GE Healthcare France (France)

\section{SESSION 12 CT ALGORITHMS}

$72581 Q \quad$ Geometric calibration and distortion calibration for CT from scans of unknown objects using complementary rays [7258-61]

K. M. Holt, Varian Medical Systems, Inc. (United States)

7258 is Optimal binary coding matrices for multiplexed x-ray imaging [7258-63]

D. S. Lalush, North Carolina State Univ. (United States) and The Univ. of North Carolina at Chapel Hill (United States)

7258 1T Quantitative evaluation of noise reduction algorithms for very low dose renal CT perfusion imaging [7258-64]

X. Liu, A. N. Primak, L. Yu, H. Li, J. D. Krier, L. O. Lerman, C. H. McCollough, Mayo Clinic (United States)

$72581 \mathrm{U}$ Prior-image-based few-view cone beam CT for applications to daily scan in image-guided radiation therapy: preliminary study [7258-65]

S. Cho, E. Pearson, E. Y. Sidky, J. Bian, C. A. Pelizzari, X. Pan, The Univ. of Chicago (United States) 
$72581 \mathrm{~V}$ Image-based online correction of misalignment artifacts in cone-beam CT [7258-66] Y. Kyriakou, Friedrich-Alexander-Univ. Erlangen-Nuremberg (Germany); R. M. Lapp, VAMP GmbH (Germany); L. Hillebrand, D. Ertel, W. A. Kalender, Friedrich-Alexander-Univ. Erlangen-Nuremberg (Germany)

7258 IW Image-based iterative compensation of motion artifacts in computed tomography [7258-67]

C. Schretter, Otto-von-Guericke-Univ. Magdeburg (Germany) and Philips Research (Germany); C. Neukirchen, Philips Research (Germany); G. Rose, Otto-von-Guericke-Univ. Magdeburg (Germany); M. Bertram, Philips Research (Germany)

\section{Part Two}

$72581 \mathrm{X} \quad$ Elimination of blooming artifacts off stents by dual energy CT [7258-116]

Y. Zou, M. D. Silver, Toshiba Medical Research Institute USA (United States)

7258 IY Spurious structures created by interpolation-based CT metal artifact reduction [7258-69] J. Müller, T. M. Buzug, Univ. zu Lübeck (Germany)

725812 Efficient scatter correction using asymmetric kernels [7258-70]

J. Star-Lack, M. Sun, Varian Medical Systems, Inc. (United States); A. Kaestner, R. Hassanein, Varian Medical Systems AG. (Switzerland); G. Virshup, Varian Medical Systems, Inc. (United States); T. Berkus, M. Oelhafen, Varian Medical Systems AG (Switzerland)

725820 Scatter correction for x-ray conebeam CT using one-dimensional primary modulation [7258-71]

L. Zhu, H. Gao, N. R. Bennett, L. Xing, R. Fahrig, Stanford Univ. (United States)

\section{SESSION 14 CT HOT TOPICS}

725821 Optimal energy thresholds and weights for separating materials using photon counting x-ray detectors with energy discriminating capabilities [7258-72]

A. S. Wang, N. J. Pelc, Stanford Univ. (United States)

$725822 \quad$ X-ray multisource for medical imaging [7258-73] K. Frutschy, B. De Man, P. Edic, GE Global Research (United States); B. Lounsberry, GE Healthcare (United States); B. Neculaes, Y. Zou, L. Inzinna, K. Conway, X. Zhang, Y. Cao, S. Gunturi, A. Caiafa, J. Reynolds, GE Global Research (United States)

725823 SNR efficient 3D reconstruction algorithm for multi-source inverse geometry CT system [7258-74]

J. Baek, N. J. Pelc, Stanford Univ. (United States) 
725824 Characterization of a novel photon counting detector for clinical CT: count rate, energy resolution, and noise performance [7258-75]

W. C. Barber, DxRay, Inc. (United States); E. Nygard, DxRay Inc. (United States) and Interon AS (Norway); J. S. Iwanczyk, DxRay, Inc. (United States); M. Zhang, E. C. Frey, B. M. W. Tsui, The Johns Hopkins Univ. (United States); J. C. Wessel, Interon AS (Norway); N. Malakhov, DxRay Inc. (United States) and Interon AS (Norway); G. Wawrzyniak, Interon AS (Norway); N. E. Hartsough, T. Gandhi, DxRay, Inc. (United States); K. Taguchi, The Johns Hopkins Univ. (United States)

725825 Photon-counting CT with silicon detectors: feasibility for pediatric imaging [7258-76] M. Yveborg, C. Xu, E. Fredenberg, M. Danielsson, Royal Institute of Technology (Sweden)

\section{SESSION 15 CT RECONSTRUCTION}

725826 Performance comparison between compressed sensing and statistical iterative reconstruction algorithms [7258-77]

J. Tang, B. Nett, G.-H. Chen, Univ. of Wisconsin, Madison (United States)

725827 Boundary reconstruction in limited-angle x-ray phase-contrast tomography [7258-78] M. A. Anastasio, Illinois Institute of Technology (United States); E. Y. Sidky, X. Pan, The Univ. of Chicago (United States); C.-Y. Chou, National Taiwan Univ. (Taiwan)

725828 A dual formulation of a penalized maximum likelihood x-ray CT reconstruction problem [7258-79]

J. Xu, K. Taguchi, The Johns Hopkins Univ. (United States); G. T. Gullberg, Lawrence Berkeley National Lab. (United States); B. M. W. Tsui, The Johns Hopkins Univ. (United States)

725829 Noise properties of iterative reconstruction techniques in low-dose CT scans [7258-80] S. Do, M. K. Kalra, Massachusetts General Hospital (United States) and Harvard Medical School (United States); Z. Liang, Univ. of California, Los Angeles (United States); W. C. Karl, Boston Univ. (United States); T. J. Brady, H. Pien, Massachusetts General Hospital (United States) and Harvard Medical School (United States)

7258 2A Interactive GPU-accelerated image reconstruction in cone-beam CT [7258-81] L. Hillebrand, Friedrich-Alexander-Univ. Erlangen-Nürnberg (Germany); R. M. Lapp, VAMP GmbH (Germany); Y. Kyriakou, W. A. Kalender, Friedrich-Alexander-Univ. Erlangen-Nürnberg (Germany)

7258 2B GPU-accelerated SART reconstruction using the CUDA programming environment [7258-82] B. Keck, Friedrich-Alexander-Univ. Erlangen-Nuremberg (Germany) and Siemens Healthcare, (Germany); H. Hofmann, Friedrich-Alexander-Univ. Erlangen-Nuremberg (Germany); H. Scherl, M. Kowarschik, Siemens Healthcare, (Germany); J. Hornegger, Friedrich-Alexander-Univ. Erlangen-Nürnberg (Germany)

\section{POSTER SESSION: ALGORITHMS}

7258 2C Effect of detector time delay and its correction in multiple source computed tomography [7258-83]

A. Thran, P. Forthmann, R. Proksa, Philips Research (Germany) 
7258 2D Noise correlation in CBCT projection data and its application for noise reduction in low-dose CBCT [7258-84]

J. Wang, Stanford Univ. School of Medicine (United States);A. Chai, Stanford Univ. School of Medicine (United States) and Stanford Univ. (United States); L. Xing, Stanford Univ. School of Medicine (United States)

$72582 \mathrm{E} \quad$ Geometric calibration of detectors with discrete irregularities for computed tomography (Honorable Mention Poster Award) [7258-85]

K. M. Holt, Varian Medical Systems, Inc. (United States)

7258 2F Simultaneous activity and attenuation reconstruction in PET: applying partially known attenuation [7258-86]

S. S. Furuie, Univ São Paulo (Brazil)

$72582 \mathrm{H} \quad$ Real-time compression of raw computed tomography data: technology, architecture, and benefits [7258-88]

A. Wegener, Samplify Systems (United States); N. Chandra, GE Healthcare (United States);

Y. Ling, Samplify Systems (United States); R. Senzig, GE Healthcare (United States);

R. Herfkens, Stanford Univ. School of Medicine (United States)

$725821 \quad$ Perfusion from angiogram and a priori (PAP) with temporal regularization [7258-89]

K. Taguchi, J.-F. H. Geschwind, The Johns Hopkins Univ. School of Medicine (United States)

7258 2J Bronchial wall region extraction algorithm using multi-slice CT images [7258-90]

K. Akashi, S. Saita, M. Kubo, Y. Kawata, N. Niki, Univ. of Tokushima (Japan); Y. Nakano, Shiga Univ. School of Medicine (Japan); A. Niimi, H. Matsumoto, T. Oguma, Kyoto Univ. Graduate School of Medicine (Japan); Y. Takiguchi, N. Kawata, N. Tanabe, Chiba Univ. Graduate School of Medicine (Japan); H. Ohmatsu, National Cancer Ctr. Hospital East(Japan); K. Eguchi, Teikyo Univ. School of Medicine (Japan); M. Kaneko, National Cancer Ctr. (Japan); N. Moriyama, National Cancer Ctr. Research Ctr. for Cancer Prevention and Screening(Japan)

7258 2K Volumetric soft tissue brain imaging on xCAT, a mobile flat-panel x-ray CT system [7258-91] W. Zbijewski, Xoran Technologies, Inc. (United States); J. W. Stayman, Xoran Technologies, Inc. (United States) and Michigan Tech Research Institute (United States)

$72582 \mathrm{~L} \quad$ Image reconstruction for a stationary digital breast tomosynthesis system [7258-92] R. Rajaram, G. Yang, The Univ. of North Carolina at Chapel Hill (United States); E. Quan, B. Frederick, D. S. Lalush, North Carolina State Univ. (United States) and The Univ. of North Carolina at Chapel Hill (United States); O. Z. Zhou, The Univ. of North Carolina at Chapel Hill (United States)

7258 2M Local correction of non-periodic motion in computed tomography [7258-93] C. Schretter, Otto-von-Guericke-Univ. Magdeburg (Germany) and Philips Research Europe (Germany); C. Neukirchen, Philips Research Europe (Germany); G. Rose, Otto-vonGuericke-Univ. Magdeburg (Germany); M. Bertram, Philips Research Europe (Germany)

$72582 \mathrm{~N} \quad$ Toward region of interest computer tomography [7258-94]

S. Schafer, P. B. Noël, A. M. Walczak, K. R. Hoffmann, Univ. at Buffalo (United States) and Toshiba Stroke Research Ctr., Univ. at Buffalo (United States) 
725820 Wavelet based de-noising methods for local SPECT reconstruction with nonuniform attenuation [7258-95]

L. Wang, J. Wen, J. Yang, Y. Chen, Beijing Institute of Technology (China); Z. Liang, Stony

Brook Univ. (United States)

7258 2P A noise decomposition method for image quality analysis of medical radiography detectors [7258-96]

S. Al Tahli, I. Maack, A. Koch, C. Herrmann, Philips Medical Systems DMC GmbH (Germany)

$72582 \mathrm{E} \quad$ Evaluating low pass filters on SPECT reconstructed cardiac orientation estimation [7258-97]

S. Dwivedi, Philips Healthcare (India)

POSTER SESSION: APPLICATIONS

$72582 R \quad$ Systematic scanner variability of patient CT attenuation measurements [7258-98]

P. F. Judy, R. D. Nawfel, S. G. Silverman, Brigham and Women's Hospital (United States) and Harvard Medical School (United States)

$725825 \quad$ An experimental cone-beam micro-CT system for small animal imaging [7258-99]

S. Zhu, J. Tian, G. Yan, C. Qin, J. Liu, Institute of Automation (China)

7258 2T 4D micro-CT-based perfusion imaging in small animals (Cum Laude Poster Award) [7258-100]

C. T. Badea, S. M. Johnston, M. Lin, L. W. Hedlund, G. A. Johnson, Duke Univ. Medical Ctr. (United States)

$72582 \mathrm{U} \quad$ Aftenuation and image noise level based online z-axis tube current modulation for CT scans independent with localizer radiograph: simulation study and results [7258-101]

Y. Tian, M. Chen, J. Kong, Siemens Shanghai Medical Equipment Ltd. (China)

$72582 \mathrm{~V} \quad \mathrm{CBCT} / \mathrm{CBDT}$ equipped with the $\mathrm{x}$-ray projection system for image-guided proton therapy [7258-102]

M. K. Cho, Pusan National Univ. (Korea, Republic of); J. S. Kim, National Cancer Ctr. (Korea, Republic of); Y.-B. Cho, Princess Margaret Hospital (Canada); H. Youn, Pusan National Univ. (Korea, Republic of); S. Y. Park, National Cancer Ctr. (Korea, Republic of); S. Cho, The Univ. of Chicago (United States); H. K. Kim, Pusan National Univ. (Korea, Republic of)

7258 2W Clinical micro-CT for dental imaging [7258-103]

H. Youn, M. K. Cho, C.-S. Shon, Pusan National Univ. (Korea, Republic of); B. H. Cho, Pusan National Univ. School of Dentistry (Korea, Republic of); C. H. Kim, Vatech Co., Ltd. (Korea, Republic of); H. K. Kim, Pusan National Univ. (Korea, Republic of)

\section{POSTER SESSION: BREAST IMAGING}

$72582 Y \quad$ Preliminary feasibility of dedicated breast CT with an inverse geometry [7258-106]

T. Gilat Schmidt, Marquette Univ. (United States)

$72582 Z$ Study of signal-to-noise ratio in digital mammography [7258-107]

Y. Kato, N. Fujita, Graduate School of Medical Sciences, Nagoya Univ. (Japan); Y. Kodera, School of Health Sciences, Nagoya Univ. (Japan) 
$725830 \quad$ Imaging technique optimization of tungsten anode FFDM system [7258-108]

B. Chen, A. P. Smith, Z. Jing, E. Ingal, Hologic, Inc. (United States)

725831 Digital breast tomosynthesis (DBT) versus full field digital mammography (FFDM): comparison of a system performance using a contrast detail phantom [7258-109] A. Nitrosi, G. Borasi, M. Bertolini, A. Botti, D. Tassoni, Arcispedale S. Maria Nuova (Italy); S. Strocchi, Azienda Ospedale di Circolo e Fondazione Macchi (Italy)

\section{POSTER SESSION: CARDIAC IMAGING}

725832 Cardiac imaging with multi-sector data acquisition in volumetric CT: variation of effective temporal resolution and its potential clinical consequences [7258-1 10] X. Tang, J. Hsieh, B. H. Taha, M. L. Vass, J. L. Seamans, D. R. Okerlund, GE Healthcare (United States)

725833 Advanced processing for motion-compensated reconstruction in cardiac cone-beam CT (Honorable Mention Poster Award) [7258-111]

U. van Stevendaal, P. Forthmann, T. Köhler, J. von Berg, C. Lorenz, M. Grass, Philips Research Labs. Hamburg (Germany)

725834 A second pass gated reconstruction scheme with conjugate weights [7258-112] K. Erhard, M. Grass, Philips Research (Germany)

725835 A cardiac phantom study on quantitative correction of coronary calcium score on multi-detector, dual source, and electron beam tomography for velocity, calcification density, and acquisition time [7258-113]

M. J. W. Greuter, J. M. Groen, L. J. Nicolai, H. Dijkstra, M. Oudkerk, Univ. Medical Ctr. Groningen (Netherlands)

725836 Diagnostic quality of time-averaged ECG-gated CT data [7258-114]

A. Klein, Institute of Technical Medicine, Univ. Twente (Netherlands); L. J. Oostveen, Radboud Univ. Nijmegen Medical Ctr. (Netherlands); M. J. W. Greuter, Univ. Medical Ctr. Groningen (Netherlands); Y. Hoogeveen, L. J. Schultze Kool, Radboud Univ. Nijmegen Medical Ctr. (Netherlands); C. H. Slump, Institute of Technical Medicine, Univ. Twente (Netherlands); W. K. J. Renema, Radboud Univ. Nijmegen Medical Ctr. (Netherlands)

\section{POSTER SESSION: CT CORRECTIONS}

725837 An online cross-scatter correction algorithm for dual-source CT: effects on CT number accuracy and noise [7258-115]

C. D. Eusemann, A. Apel, B. Schmidt, Siemens Healthcare (Germany); A. I. Walz-Flannigan, M. C. Jacobsen, Mayo Clinic (United States); K. Stierstorfer, T. G. Flohr, Siemens Healthcare (Germany); C. H. McCollough, Mayo Clinic (United States)

725838 Iterative scatter correction for $x$-ray cone-beam CT with semi-transparent beam stop array [7258-117]

H. Yan, X. Mou, S. Tang, X. Chen, Xi'an Jiaotong Univ. (China)

725839 Analytical scatter estimation for cone-beam computed tomography [7258-1 18] H. R. Ingleby, I. A. Elbakri, D. W. Rickey, S. Pistorius, CancerCare Manitoba (Canada) 
7258 3A High-contrast artifact reduction in cone beam computed tomography by using geometric techniques [7258-119]

P. B. Noël, Univ. at Buffalo (United States) and Toshiba Stroke Research Ctr., Univ. at Buffalo (United States); J. XU, Toshiba Stroke Research Ctr., Univ. at Buffalo (United States);

K. R. Hoffmann, Univ. at Buffalo (United States) and Toshiba Stroke Research Ctr., Univ. at Buffalo (United States); J. J. Corso, Univ. at Buffalo (United States); S. Schafer, A. M. Walczak, Toshiba Stroke Research Ctr., Univ. at Buffalo (United States)

7258 3B Real time implementation of distortion corrections for a tiled EMCCD-based solid state $\mathrm{x}$-ray image intensifier (SSXII) [7258-120]

C. Keleshis, K. R. Hoffmann, J. Lee, H. Hamwi, W. Wang, C. N. Ionita, D. R. Bednarek,

A. Verevkin, S. Rudin, Toshiba Stroke Research Ctr., Univ. at Buffalo (United States)

7258 3C Measurement-based scatter correction for cone-beam CT in radiation therapy [7258-122]

L. Zhu, L. Xing, Stanford Univ. (United States)

7258 3D Three-dimensional metal artifact reduction method for dental conebeam CT scanners [7258-123]

K. Kobayashi, A. Katsumata, Yamatake Corp. (Japan); K. Ito, T. Aoki, Tohoku Univ. (Japan)

7258 3E Reduction of artifacts due to multiple metallic objects in computed tomography [7258-124]

K. Y. Jeong, J. B. Ra, Korea Advanced Institute of Science and Technology (Korea, Republic of)

7258 3F Reduction of aliasing artifacts in tomographic images [7258-125]

O. Tischenko, Helmholtz Zentrum München GmbH (Germany); Y. Xu, Univ. of Oregon (United States); T. Goetzfried, L. Bogner, Univ. of Regensburg (Germany); C. Hoeschen, Helmholtz Zentrum München GmbH (Germany)

$72583 G \quad$ Attenuation compensation in mesh-domain OSEM SPECT reconstruction [7258-126] L. Vogelsang, Y. Lu, Syracuse Univ. (United States); B. Yu, China Three Gorges Univ. (China); A. Krol, Upstate Medical Univ., SUNY (United States) and Syracuse Univ. (United States); Y. XU, X. Hu, Syracuse Univ. (United States); D. Feiglin, Upstate Medical Univ., SUNY (United States) and Syracuse Univ. (United States); E. Lipson, Syracuse Univ. (United States) and Upstate Medical Univ., SUNY (United States)

$72583 \mathrm{H} \quad$ How many $x$-ray photons can be scattered from a SPECT/CT room to an adjacent gamma camera? [7258-127]

Z. Cao, Medical College of Georgia (United States)

POSTER SESSION: DETECTORS

$725831 \quad$ Feasibility study of CMOS detectors for mammography [7258-40]

J. C. Han, S. Yun, C. H. Lim, Pusan National Univ. (Korea, Republic of); T. W. Kim, Vatech Co., Ltd. (Korea, Republic of); H. K. Kim, Pusan National Univ. (Korea, Republic of)

7258 3J Flat detector ghost image reduction by UV irradiation [7258-128]

R. M. Snoeren, H. Steinhauser, L. Alving, H. Stouten, Philips Healthcare (Netherlands);

P. H. N. de With, Univ. of Technology Eindhoven (Netherlands) 
7258 3K A counting and integrating pixel readout chip for amorphous selenium direct radiation detectors for medical imaging applications (Honorable Mention Poster Award) [7258-129]

A. H. Goldan, B. Hadji, K. S. Karim, Univ. of Waterloo (Canada); G. DeCrescenzo,

J. A. Rowlands, Thunder Bay Regional Research Institute, Lakehead Univ. (Canada);

O. Tousignant, L. Laperrière, Anrad Corp. (Canada)

7258 3L Quasi-monochromatic x-ray filter with thin film multilayer for a large area radiation field [7258-130]

Y. Park, S. Han, J. Chae, C. Kim, Hanwha L\&C Research Ctr. (Korea, Republic of); K. S. Chon, Wonkwang Univ. (Korea, Republic of); H.-K. Lee, College of Medicine, The Catholic Univ. of Korea (Korea, Republic of); D. S. Han, Monoray Co., Ltd (Korea, Republic of)

7258 3M Advanced a-Se film with high sensitivity and heat resistance for $\mathrm{x}$-ray detectors (Honorable Mention Poster Award) [7258-131]

K. Ogusu, O. Nakane, Y. Igasaki, Y. Okamura, S. Yamada, T. Hirai, Hamamatsu Photonics K.K. (Japan)

$72583 \mathrm{~N}$ Pixel-structured scintillators for digital x-ray imaging (Honorable Mention Poster Award) [7258-133]

S. M. Yun, C. H. Lim, Pusan National Univ. (Korea, Republic of); T. W. Kim, Vatech Co., Ltd. (Korea, Republic of); H. K. Kim, Pusan National Univ. (Korea, Republic of)

725830 Optimized operation and offset corrections for a battery-powered wireless digital x-ray detector [7258-135]

K. Töpfer, J. Dehority, Carestream Health, Inc. (United States)

7258 3P Betacam: a commercial approach to $\beta$-autoradiography [7258-136]

J. Cabello, Univ. of Surrey (United Kingdom); A. Holland, K. Holland, XCAM Ltd. (United

Kingdom); A. Bailey, I. Kitchen, K. Wells, Univ. of Surrey (United Kingdom)

$72583 Q \quad$ Characterization of bias induced metastability of amorphous silicon thin film transistor based passive pixel sensor switch and its impact on biomedical x-ray imaging application [7258-137]

A. Sultana, N. Safavian, M. H. Izadi, K. S. Karim, Univ. of Waterloo (Canada); J. A. Rowlands, Univ. of Waterloo (Canada) and Thunder Bay Regional Research Institute, Lakehead Univ. (Canada)

\section{POSTER SESSION: DUAL ENERGY}

7258 3R Dual energy with dual source CT and kVp switching with single source CT: a comparison of dual energy performance [7258-138]

M. Grasruck, S. Kappler, M. Reinwand, K. Stierstorfer, Siemens AG (Germany)

725835 Image-based dual energy CT improvements using Gram-Schmidt method [7258-139]

K.-K. Park, Arizona State Univ. (United States); W. Pavlicek, T. Boltz, R. Paden, A. Hara, Mayo Clinic (United States); M. Akay, Arizona State Univ. (United States)

7258 3T Dual energy CT via fast kVp switching spectrum estimation [7258-140]

D. XU, D. A. Langan, X. WU, J. D. Pack, T. M. Benson, J. E. Tkaczky, A. M. Schmitz, GE Global Research (United States) 
$72583 \mathrm{U}$ Dual-energy contrast enhanced digital mammography: theoretical and experimental study of optimal monoenergetic beam parameters using synchrotron radiation [7258-141] S. Puong, R. lordache, X. Bouchevreau, S. Muller, GE Healthcare France (France)

7258 3V Pre-reconstruction three-material decomposition in dual-energy CT [7258-142] L. Yu, X. Liu, C. H. McCollough, Mayo Clinic (United States)

7258 3W Enhanced discrimination of calcified and soft arterial plaques using computed tomography with a multi-energy-window photon counting $\mathbf{x}$-ray detector [7258-143]

X. Wang, J. XU, K. Taguchi, The Johns Hopkins Univ. (United States); B. E. Patt, D. J. Wagenaar, Gamma Medica-Ideas, Inc. (United States); E. C. Frey, The Johns Hopkins Univ. (United States)

\section{Part Three}

7258 3X Impact of scattered radiation on spectral CT [7258-144] J. Wiegert, K. J. Engel, C. Herrmann, Philips Research (Germany)

7258 3Y Empirical projection-based basis-component decomposition method [7258-145] B. Brendel, E. Roessl, J.-P. Schlomka, R. Proksa, Philips Research (Germany)

$72583 Z$ Linearity between CT number and iodine concentration and application to improving accuracy of CT number in slow kV-switching dual energy CT [7258-146] Y. Zou, M. D. Silver, Toshiba Medical Research Institute USA (United States)

725840 Scatter correction algorithm without extra exposure for dual-energy digital mammography [7258-147]

X. Chen, X. Mou, H. Yan, Xi'an Jiaotong Univ. (China); H. Yu, Virginia Polytechnic Institute and State Univ. (United States); L. Zhang, The Hong Kong Polytechnic Univ. (Hong Kong, China)

$725841 \quad$ A material decomposition method for dual energy micro-CT [7258-148] S. M. Johnston, G. A. Johnson, C. T. Badea, Duke Univ. Medical Ctr. (United States)

725842 Dual-energy performance of dual $\mathrm{kVp}$ in comparison to dual-layer and quantum-counting CT system concepts [7258-149]

S. Kappler, M. Grasruck, D. Niederlöhner, M. Strassburg, S. Wirth, Siemens AG (Germany)

$725843 \quad$ Fast kVp switching CT imaging of a dynamic cardiac phantom [7258-150] J. D. Pack, D. A. Langan, X. WU, D. XU, T. M. Benson, A. M. Schmitz, J. E. Tkaczyk, GE Global Research (United States); W. Pavlicek, T. F. Boltz II, R. Payden, Mayo Clinic Scottsdale (United States); J. Leverentz, P. Licato, GE Healthcare (United States)

725844 Advanced material separation technique based on dual energy CT scanning [7258-151] A. A. Zamyatin, A. Natarajan, Y. Zou, Toshiba Medical Research Institute USA (United States)

725845 Monochromatic CT image representation via fast switching dual kVp [7258-152] X. Wu, D. A. Langan, D. XU, T. M. Benson, J. D. Pack, A. M. Schmitz, E. J. Tkaczyk, GE Global Research (United States); J. Leverentz, P. Licato, GE Healthcare (United States) 
725846 Development of a high-speed CT imaging system using EMCCD camera [7258-153]

S. C. Thacker, Radiation Monitoring Devices, Inc. (United States); K. Yang, N. Packard, Univ. of California, Davis Medical Ctr. (United States); V. Gaysinskiy, Radiation Monitoring Devices, Inc. (United States); G. Burkett, Univ. of California, Davis Medical Ctr. (United States); S. Miller, Radiation Monitoring Devices, Inc. (United States); J. M. Boone, Univ. of California, Davis Medical Ctr. (United States); V. Nagarkar, Radiation Monitoring Devices, Inc. (United States)

725847 Stationary micro-CT scanner using a distributed multi-beam field emission x-ray source: a feasibility study [7258-154]

R. Peng, J. Zhang, X. Calderon-Colon, S. Wang, S. Sultana, S. Chang, J. P. Lu, The Univ. of North Carolina at Chapel Hill (United States); O. Zhou, The Univ. of North Carolina at Chapel Hill (United States) and Lineberger Comprehensive Cancer Ctr. (United States)

725848 Liquid lens confocal microscopy with advanced signal processing for higher resolution 3D imaging [7258-155]

N. A. Riza, M. A. Sheikh, College of Optics \& Photonics, Univ. of Central Florida (United States)

7258 4A A novel method to reduce data acquisition time in differential phase contrast: computed tomography using compressed sensing [7258-157]

Z. Qi, J. Zambelli, N. Bevins, G.-H. Chen, Univ. of Wisconsin, Madison (United States)

7258 4B Investigation of quantitative polychromatic $x$-ray phase-contrast tomography for tissue characterization [7258-158]

C.-Y. Chou, National Taiwan Univ. (Taiwan); A. M. Zysk, M. A. Anastasio, Illinois Institute of Technology (United States)

7258 4C MEG beamforming: magnetic source imaging [7258-159]

T. Lei, T. P. L. Roberts, Children's Hospital of Philadelphia (United States) and Univ. of Pennsylvania (United States)

7258 4D Dose efficiency consideration for volume-of-interest breast imaging using $\mathbf{x}$-ray differential phase-contrast CT [7258-161]

W. Cai, R. Ning, Univ. of Rochester (United States)

7258 4E Analysis of the spectrum in phase-contrast mammography [7258-163]

A. M. Zysk, Q. Xu, L. de Sisternes, J. G. Brankov, M. N. Wernick, M. A. Anastasio, Illinois Institute of Technology (United States)

7258 4G Three-dimensional photoacoustic tomography of finger joint: from phantom experiment to in vivo study [7258-165]

Y. Sun, H. Jiang, Univ. of Florida (United States) 
$72584 \mathrm{H}$ Marginal adaptation analysis performed with en face optical coherence tomography in fixed partial dentures [7258-166]

C. Sinescu, M. L. Negrutiu, S. Antonie, Univ. of Medicine and Pharmacy Victor Babes Timisoara (Romania); G. Dobre, A. Bradu, M. Hughes, Univ. of Kent (United Kingdom); M. Rominu, Univ. of Medicine and Pharmacy Victor Babes Timisoara (Romania);

A. Gh. Podoleanu, Univ. of Kent (United Kingdom)

$725841 \quad$ Resolution improvement of the molecular imaging technique based on magnetic nanoparticles [7258-167]

Y. Ishihara, Y. Kusayama, Nagaoka Univ. of Technology (Japan)

$7258 \mathrm{4J}$ Method for optic nerve intensity characterization in mice from $\mathbf{M n}^{2+}$ enhanced $\mathrm{MR}$ images [7258-168]

D. Xiao, The Australian E-Health Research Ctr., ICTC, CSIRO (Australia); J. Ribic, The Univ. of Melbourne (Australia) and Univ. of Oslo (Norway); H. Butzkueven, K. Fang, T. J. Kilpatrick, G. F. Egan, The Univ. of Melbourne (Australia); O. Salvado, The Australian E-Health Research Ctr., ICTC, CSIRO(Australia)

$72584 \mathrm{~K}$ Effect of masticatory load on cracks deflection/penetration investigated with en face optical coherence tomography in ceramic fixed partial dentures [7258-169]

C. Sinescu, M. L. Negrutiu, Univ. of Medicine and Pharmacy Victor Babes Timisoara (Romania); L. Marsavina, R. Negru, M. Hluscu, C. Caplescu, Politehnica Univ. Timisoara (Romania); A. Bradu, M. Hughes, Univ. of Kent (United Kingdom); M. Rominu, Univ. of Medicine and Pharmacy Victor Babes Timisoara (Romania); A. Gh. Podoleanu, Univ. of Kent (United Kingdom)

$72584 \mathrm{~L} \quad$ Effect of subdicing on the dispersion and resonance behavior of elastic guided waves in 1D array ultrasound transducers [7258-170]

D. H. Cortes, West Virginia Univ. (United States); S. K. Datta, Univ. of Colorado at Boulder (United States); O. M. Mukdadi, West Virginia Univ. (United States)

7258 4M Image quality and signal distribution in 1.5-T and 3-T MRI in mild traumatic brain injury patients [7258-171]

M. Rossi, Tampere Univ. Hospital (Finland); P. Dastidar, Tampere Univ. Hospital (Finland) and Tampere Medical School (Finland); P. Ryymin, A. Ylinen, J. Öhman, Tampere Univ. Hospital (Finland); S. Soimakallio, Tampere Univ. Hospital (Finland) and Tampere Medical School (Finland); H. Eskola, Tampere Univ. Hospital (Finland) and Tampere Univ. of Technology (Finland)

$72584 \mathrm{~N}$ Optical coherence tomography and confocal microscopy investigations of dental structures and restoration materials [7258-172]

M. L. Negrutiu, C. Sinescu, M. Rominu, Univ. of Medicine and Pharmacy Victor Babes Timisoara (Romania); M. Hughes, G. Dobre, A. Gh. Podoleanu, Univ.of Kent (United Kingdom)

725840 A new linear least squares method for T1 estimation from SPGR signals with multiple TRs [7258-173]

L.-C. Chang, The Catholic Univ. of America (United States) and National Institutes of Health

(United States); C. G. Koay, P. J. Basser, C. Pierpaoli, National Institutes of Health (United States) 
POSTER SESSION: PERFORMANCE

7258 4P Image quality evaluation of direct-conversion digital mammography system with new dual a-Se layer detector [7258-174]

T. Kuwabara, N. Iwasaki, T. Sendai, R. Furue, T. Agano, FUJIFILM Corp. (Japan)

$72584 Q \quad$ The effect of dose reductions on lesion detection in head CT [7258-175]

S. Elojeimy, W. Huda, Medical Univ. of South Carolina (United States); K. M. Ogden, SUNY Upstate Medical Univ. (United States); R. Owen, Medical Univ. of South Carolina (United States); E. Samei, Duke Univ. Medical Ctr. (United States); Z. Rumboldt, Medical Univ. of South Carolina (United States)

7258 4R Modification of the NEMA XR21-2000 cardiac phantom for testing of imaging systems used in endovascular image guided interventions [7258-176]

C. N. Ionita, A. Dohatcu, A. Jain, C. Keleshis, K. R. Hoffmann, D. R. Bednarek, S. Rudin, Toshiba Stroke Research Ctr., Univ. at Buffalo (United States) and Univ. at Buffalo (United States)

7258 4S Image quality evaluation of a LightSpeed CT750 HD computed tomography system [7258-177]

J. Fan, F. Dong, P. Sainath, J. Hsieh, X. Tang, T. Toth, B. Li, P. Crandall, R. Senzig, A. Dixon, GE Healthcare (United States)

7258 4T Computed tomography quality indexes: evaluation experience [7258-178]

S. Strocchi, C. Vite, Ospedale di Circolo e Fondazione Macchi (Italy); R. Novario, Insubria Univ. (Italy); M. Cacciatori, G. Frigerio, Sant' Anna Hospital (Italy); L. Conte, Insubria Univ. (Italy)

$72584 \mathrm{U} \quad$ Optimizing image quality using statistical multivariate optimization methodology using desirability functions [7258-179]

D. L. Leong, Analogic Corp. (United States) and Univ. College Dublin School of Medicine and Medical Sciences (Ireland); P. C. Brennan, Univ. College Dublin School of Medicine and Medical Sciences (Ireland)

7258 4V GATE simulations of CTDI for patient dose [7258-180]

C.-L. Lee, H.-J. Kim, H.-M. Cho, H.-S. Park, A-R. Yoo, Yonsei Univ. (Korea, Republic of)

$72584 \mathrm{Y}$ Contrast-detail comparison between unprocessed and processed CDMAM images [7258-184]

F. Zanca, H. Bosmans, J. Jacobs, K. Michielsen, F. Sisini, J. Nens, Univ. Hospital Gasthuisberg

(Belgium); K. C. Young, The Royal Surrey County Hospital NHS Trust (United Kingdom);

E. Shaheen, A. Jacobs, G. Marchal, Univ. Hospital Gasthuisberg (Belgium)

$72584 Z$ A simple method for evaluating image quality of screen-film system using a highperformance digital camera [7258-186]

N. Fujita, Graduate School of Medical Sciences, Nagoya Univ. (Japan); A. Yamazaki, Osaka General Medical Ctr. (Japan); K. Ichikawa, Graduate School of Medical Sciences, Kanazawa Univ. (Japan); Y. Kodera, School of Health Sciences, Nagoya Univ. (Japan)

725850 Image quality of a cone beam O-arm 3D imaging system [7258-187]

J. Zhang, V. Weir, J. Lin, H. Hsiung, E. R. Ritenour, Univ. of Minnesota (United States) 
725852 MTF compensation for digital radiography system with indirect conversion flat panel detector [7258-189]

W. Zhang, GE Global Research (China); H. Souchay, GE Healthcare France (France);

C. Yang, GE Global Research (China)

\section{POSTER SESSION: RECONSTRUCTION}

725854 Tomographic mesh generation for OSEM reconstruction of SPECT images [7258-191]

Y. Lu, Syracuse Univ. (United States); B. YU, China Three Gorges Univ. (China); L. Vogelsang,

Syracuse Univ. (United States); A. Krol, SUNY Upstate Medical Univ. (United States) and Syracuse Univ. (United States); Y. XU, X. Hu, Syracuse Univ. (United States); D. Feiglin, SUNY Upstate Medical Univ. (United States) and Syracuse Univ. (United States)

725855 Dose reduction in digital breast tomosynthesis using a penalized maximum likelihood reconstruction [7258-193]

M. Das, H. Gifford, M. O'Connor, S. J. Glick, Univ. of Massachusetts Medical School (United States)

725856 Implementation of an effective KL domain penalized weighted least-squares sinogram restoration for low-dose CT colonography [7258-194]

Y. Fan, J. Wang, Stony Brook Univ. (United States); H. Lu, Fourth Military Medical Univ. (China); Z. Liang, Stony Brook Univ. (United States)

725857 Spatio-temporal filtration of dynamic CT data using diffusion filters [7258-195]

H. Bruder, R. Raupach, E. Klotz, K. Stierstorfer, T. Flohr, Siemens, HealthCare (Germany)

725858 RGBA packing for fast cone beam reconstruction on the GPU [7258-196]

F. Ino, S. Yoshida, K. Hagihara, Osaka Univ. (Japan)

725859 Breast tomosynthesis reconstruction with a multi-beam $x$-ray source [7258-197] Y. Chen, W. Zhou, Southern Illinois Univ., Carbondale (United States); G. Yang, X. Qian, The Univ. of North Carolina at Chapel Hill (United States); J. Lu, O. Zhou, Lineberger Comprehensive Cancer Ctr., The Univ. of North Carolina at Chapel Hill (United States)

7258 5A High performance tomosynthesis enabled via a GPU-based iterative reconstruction framework [7258-198]

F. XU, A. Khamene, O. Fluck, Siemens Corporate Research (United States)

7258 5B Enhancement of mobile C-arm cone-beam reconstruction using prior anatomical models [7258-199]

O. Sadowsky, J. Lee, The Johns Hopkins Univ. (United States); E. G. Sutter, S. J. Wall, International Ctr. for Orthopaedic Advancement, The Johns Hopkins Bayview Medical Ctr., (United States); J. L. Prince, R. H. Taylor, The Johns Hopkins Univ. (United States)

7258 5C Implementation OSEM mesh-domain SPECT reconstruction with explicit prior information [7258-200]

A. Krol, SUNY Upstate Medical Univ. (United States) and Syracuse Univ. (United States);

L. Vogelsang, Y. Lu, Y. XU, X. Hu, L. Shen, Syracuse Univ. (United States); D. Feiglin, SUNY Upstate Medical Univ. (United States) and Syracuse Univ. (United States); E. Lipson, Syracuse Univ. (United States) and SUNY Upstate Medical Univ. (United States) 
7258 5D A new design for a micro-CT scanner [7258-201]

M. Klaften, A. Schegerer, O. Tischenko, Helmholtz Zentrum München (Germany); Y. Xu, Univ. of Oregon (United States); T. Förster, M. Hrabé de Angelis, C. Hoeschen, Helmholtz Zentrum München (Germany)

7258 5E Local cone-beam SPECT reconstruction with non-uniform attenuation [7258-202]

Y. Chen, J. Wen, L. Wang, Beijing Institute of Technology (China); Z. Liang, Stony Brook Univ., SUNY (United States)

$72585 \mathrm{~F} \quad$ Fast implementation of the image reconstruction algorithm OPED [7258-203]

Y. XU, Univ. of Oregon (United States); O. Tischenko, C. Hoeschen, Helmholtz Zentrum

München, GmbH (Germany)

7258 5G Exact and stable interior ROI reconstruction for radial MRI [7258-204]

J. Zhang, Univ. of Minnesota (United States); H. Yu, Virginia Polytechnic Institute and State Univ. (United States); C. Corum, M. Garwood, Univ. of Minnesota (United States); G. Wang,

Virginia Polytechnic Institute and State Univ. (United States)

$72585 \mathrm{H} \quad$ Monte Carlo investigation of phosphor screen optics for use in indirect-conversion detectors [7258-205]

C. H. Lim, Pusan National Univ. (Korea, Republic of); C.-S. Shon, Vatech Co., Ltd. (Korea, Republic of); J. C. Han, Pusan National Univ. (Korea, Republic of); T. W. Kim, Vatech Co., Ltd. (Korea, Republic of); H. K. Kim, Pusan National Univ. (Korea, Republic of)

$725851 \quad$ Implementation of penalized-likelihood statistical reconstruction for polychromatic dualenergy CT [7258-206]

Q. XU, X. Mou, S. Tang, W. Hong, Y. Zhang, T. Luo, Xi'an Jiaotong Univ. (China)

7258 5J Feasibility of GPU-assisted iterative image reconstruction for mobile C-arm CT [7258-207]

Y. Pan, R. Whitaker, The Univ. of Utah (United States); A. Cheryauka, D. Ferguson, GE

Healthcare (United States)

POSTER SESSION: SYSTEMS

$72585 \mathrm{~L} \quad$ Initial investigation of novel trajectories to improve chest wall imaging in a dedicated breast computed tomography system [7258-209]

D. J. Crotty, Duke Univ. Medical Ctr. (United States) and Duke Univ. (United States);

R. L. McKinley, Zumatek Inc. (United States); P. Madhav, S. J. Cutler, M. P. Tornai, Duke Univ. Medical Ctr. (United States) and Duke Univ. (United States)

$72585 \mathrm{M} \quad \mathrm{X}$-ray tube-based diffraction enhanced imaging prototype images of full-thickness breast specimens: reader study evaluation [7258-210]

L. S. Faulconer, The Univ. of North Carolina at Chapel Hill (United States); C. Parham, Univ. of California, San Francisco (United States); D. J. Connor, Brookhaven National Lab. (United States); M. Koomen, C. Kuzmiak, D. Pavic, C. A. Livasy, E. Kim, D. Zeng, E. B. Cole, The Univ. of North Carolina at Chapel Hill (United States); Z. Zhong, Brookhaven National Lab. (United States); E. D. Pisano, The Univ. of North Carolina at Chapel Hill (United States) 
7258 5N Dual source CT (DSCT) imaging of obese patients: evaluation of CT number accuracy, uniformity, and noise [7258-211]

A. Walz-Flannigan, Mayo Clinic (United States); B. Schmidt, A. Apel, C. Eusemann, Siemens Healthcare (Germany); L. Yu, C. H. McCollough, Mayo Clinic (United States)

725850 Use of effective detective quantum efficiency to optimise radiographic exposures for chest imaging with computed radiography (Honorable Mention Poster Award) [7258-212] F. Ertan, King's College London (United Kingdom); A. Mackenzie, H. J. Urbanczyk, King's College Hospital (United Kingdom); N. T. Ranger, E. Samei, Duke Univ. (United States)

7258 5P Digital radiography system using a new direct-detection flat panel detector and its system performance [7258-213]

J.-H. Park, Choongwae Medical Corp. (Korea, Republic of); W.-S. Kang, Choongwae Medical Corp. (Korea, Republic of) and Yonsei Univ. College of Medicine (Korea, Republic of); B.-S. Shin, H.-S. Kang, Choongwae Medical Corp. (Korea, Republic of)

$72585 \mathrm{Q}$ A dynamic micro-CT scanner with a stationary mouse bed using a compact carbon nanotube field emission $x$-ray tube [7258-214]

G. Cao, X. Calderon-Colon, P. Wang, L. Burk, Y. Z. Lee, R. Rajaram, S. Sultana, The Univ. of North Carolina at Chapel Hill (United States); D. Lalush, North Carolina State Univ. (United States); J. Lu, The Univ. of North Carolina at Chapel Hill (United States); O. Zhou, Lineberger Comprehensive Cancer Ctr., The Univ. of North Carolina at Chapel Hill (United States)

7258 5R Dose reduction potential of the scanning geometry CT D'OR: a simulation study [7258-215] H. de las Heras, O. Tischenko, H. Schlattl, Helmholtz Zentrum München, GmbH (Germany); Y. Xu, Univ. of Oregon (United States); C. Hoeschen, Helmholtz Zentrum München, GmbH (Germany)

725855 Configuration of automatic exposure control on mammography units for computed radiography to match patient dose of screen film systems [7258-216]

C.-Y. J. Yang, W. Huang, Carestream Health, Inc. (United States)

$72585 \mathrm{~T} \quad$ Feasibility of using the micro $\mathrm{CT}$ imaging system as the conformal radiation therapy facility for small animals [7258-217]

S.-J. Tu, H.-L. Hsieh, T.-C. Chao, C.-C. Lee, Graduate Institute of Medical Physics and Imaging Science (Taiwan)

$72585 \mathrm{U}$ Calcification visibility study using combined high dose and low dose cone beam CT projections [7258-218]

Y. Zhong, C. J. Lai, L. Chen, T. Han, Y. Shen, X. Liu, T. Wang, S. Ge, Y. Yi, Z. You, C. C. Shaw, The Univ. of Texas M.D. Anderson Cancer Ctr. (United States)

\section{POSTERS: TOMOSYNTHESIS}

$72585 \mathrm{~V}$ Dose assessment of digital tomosynthesis in pediatric imaging [7258-219]

A. Gislason, CancerCare Manitoba (Canada); I. A. Elbakri, CancerCare Manitoba (Canada) and Univ. of Manitoba (Canada); M. Reed, The Children's Hospital of Winnipeg (Canada) 
7258 5W The CNR method in scan angle optimization of tomosynthesis and its limitations [7258-222] B. Ren, C. Ruth, Y. Zhang, A. Smith, C. Williams, B. Polischuk, Z. Jing, Hologic, Inc. (United States)

7258 5X Investigation of the dosimetry of chest tomosynthesis [7258-223]

A. Svalkvist, S. Zachrisson, L. G. Månsson, M. Båth, Univ. of Gothenburg (Sweden) and Sahlgrenska Univ. Hospital (Sweden)

$72585 Y \quad$ Effects of scatter radiation on reconstructed images in digital breast tomosynthesis [7258-224]

B. Liu, Massachusetts General Hospital (United States) and Harvard Medical School (United States); X. Li, Massachusetts General Hospital (United States)

$72585 Z$ Effect of non-isotropic detector blur on microcalcification detectability in tomosynthesis [7258-225]

I. S. Reiser, R. M. Nishikawa, B. A. Lau, The Univ. of Chicago (United States)

725860 Ghosting and its recovery mechanisms in multilayer selenium detectors for mammography [7258-226]

S. A. Mahmood, M. Z. Kabir, Concordia Univ. (Canada); O. Tousignant, H. Mani, Anrad Corp. (Canada)

ERRATUM

725862 Erratum to $72585 \mathrm{~W}$ : The CNR method in scan angle optimization of tomosynthesis and its limitations [7258-230]

B. Ren, C. Ruth, Y. Zhang, A. Smith, C. Williams, B. Polischuk, Z. Jing, Hologic, Inc. (United States)

Author Index

xxiv 


\title{
Conference Committee
}

\author{
Symposium Chairs
}

Armando Manduca, Mayo Clinic College of Medicine (United States)

Kevin R. Cleary, Georgetown University Medical Center (United States)

Conference Chairs

Ehsan Samei, Duke University (United States)

Jiang Hsieh, GE Healthcare (United States)

Program Committee

Guang-Hong Chen, University of Wisconsin, Madison (United States)

Mats E. Danielsson, Kungliga Tekniska Högskolan (Sweden)

Thomas G. Flohr, Siemens Healthcare (Germany)

Stephen J. Glick, University of Massachusetts Medical School (United States)

Christoph Hoeschen, Helmholtz Zentrum München, GmbH (Germany)

Hee-Joung Kim, Yonsei University (Korea, Republic of)

lacovos S. Kyprianou, Center for Devices and Radiological Health, U.S. Food and Drug Administration (United States)

Robert M. Nishikawa, The University of Chicago (United States)

Michael Overdick, Philips Research Laboratories (Germany)

Norbert J. Pelc, Stanford University (United States)

Jinyi Qi, University of California, Davis (United States)

John A. Rowlands, Thunder Bay Regional Research Institute (Canada)

Jeffrey H. Siewerdsen, Ontario Cancer Institute, Princess Margaret Hospital (Canada)

Katsuyuki Taguchi, The Johns Hopkins University (United States)

Bruce R. Whiting, Washington University in St. Louis (United States)

John Yorkston, Carestream Health, Inc. (United States)

\section{Session Chairs}

$1 \quad$ Keynote and CT Dose: Tribute to Bruce Hasegawa

Ehsan Samei, Duke University (United States)

Jiang Hsieh, GE Healthcare (United States)

2 CT Performance

Ehsan Samei, Duke University (United States)

Jiang Hsieh, GE Healthcare (United States) 
3 CT Applications

Norbert J. Pelc, Stanford University (United States)

4 Breast CT

John A. Rowlands, Thunder Bay Regional Research Institute (Canada)

5 Breast Tomosynthesis

Stephen J. Glick, University of Massachusetts Medical School (United States)

$6 \quad$ Nuclear Medicine

Katsuyuki Taguchi, The Johns Hopkins University (United States)

$7 \quad$ Non-X-Ray Imaging

Hee-Joung Kim, Yonsei University (Korea, Republic of)

Jinyi Qi, University of California, Davis (United States)

$8 \quad$ X-Ray Detectors

John Yorkston, Carestream Health, Inc. (United States)

9 Radiography and Mammography Performance

Christoph Hoeschen, Helmholtz Zentrum München, GmbH (Germany)

John A. Rowlands, Thunder Bay Regional Research Institute (Canada)

10 Photon-Counting and Direct-Conversion Systems

Mats E. Danielsson, Kungliga Tekniska Högskolan (Sweden)

11 Tomosynthesis

Christoph Hoeschen, Helmholtz Zentrum München, GmbH (Germany)

12 CT Algorithms

Thomas G. Flohr, Siemens Healthcare (Germany)

13 CT Corrections

Jeffrey H. Siewerdsen, Ontario Cancer Institute, Princess Margaret Hospital (Canada)

14 CT Hot Topics

Guang-Hong Chen, University of Wisconsin, Madison (United States)

15 CT Reconstruction

Bruce R. Whiting, Washington University in St. Lovis (United States) 


\section{In Memoriam}

\section{Robert F. Wagner \\ 1938-2008}

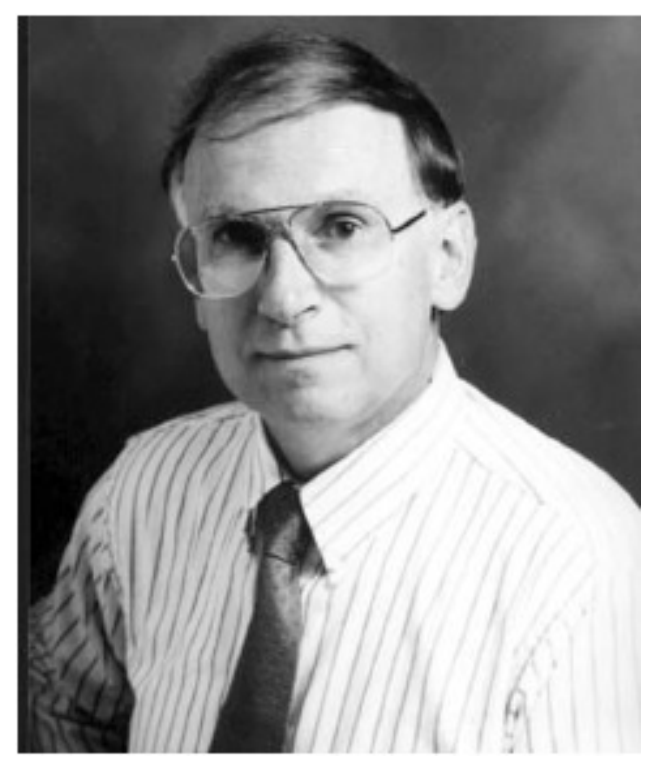

\section{A founding scientist and prolific contributor to modern medical imaging science and SPIE}

Robert F. "Bob" Wagner was a tremendous innovator in the field of medical imaging and image assessment methodologies. He was a key figure in the creation of the SPIE Medical Imaging symposium. An SPIE Fellow since 1988, Bob was active on the program committee of the Physics of Medical Imaging conference at the Medical Imaging symposium, and author of numerous technical papers published by SPIE.

"The medical imaging community has lost one of its founding fathers and most highly regarded members," said Kyle Myers, director of the Division of Imaging and Applied Mathematics at the Center for Devices and Radiological Health (CDRH), U.S. Food and Drug Administration (FDA). "Bob's career was dedicated to the development of consensus measurement methods for the assessment of medical imaging systems, quantitative medical imaging and tissue characterization, and computer-aided diagnosis. He earned an international reputation in these areas and applied his expertise to a wide range of regulatory issues central to the FDA's mission. He enlightened the scientific community within the agency as well as the international scientific community through the many invited presentations and tutorials he gave in and outside of the FDA, his numerous publications, his many professional society activities, and his assistance in regulatory decision making."

At this 2009 Medical Imaging symposium, a joint keynote session hosted by the CAD and Image Perception conferences honored Bob's many contributions from the early 1970s to the present through a series of presentations by some of his closest collaborators. 
David Brown (CDRH/FDA) recalled Bob's early years in the field, relating that after graduate and post-graduate work on the physics of nuclear interactions with radiation, Bob was hired by the Bureau of Radiological Health [a precursor to $\mathrm{CDRH}$ ] to assess the dose reduction potential of radiographic intensifying screens made with phosphors developed in the color TV industry. Within three months he published a review of the relevant imaging literature from the medical, defense, consumer, and scientific communities, together with a charter for a laboratory program. Soon after, Bob introduced digital noise analysis to radiography, and showed that the new technology offered a 1.6- to 2.5-fold exposure reduction without compromising imaging performance. He then launched a program of inter-laboratory comparison of measurements on radiographic film samples that were circulated among fifteen commercial, government, and academic laboratories worldwide. In the process he became the prime mover for work toward consensus methodology for quantitative imaging performance measurements.

Mike Insana (Univ. of Illinois at Urbana-Champaign) shared memories of his years as Bob's post-doctoral student, working with Bob on the statistical characterization of ultrasound images. He described Bob as an exemplary mentor who shared his passion and joy for science.

Myers agreed, "Bob's greatest legacy may be the many young scientists he nurtured, who either worked directly under his tutelage at the FDA or otherwise benefitted from his unfailing patience and unselfish ease of availability."

Harry Barrett (Univ. of Arizona) began his presentation by relating noise-equivalent quanta (NEQ) - a concept central to Bob's unified approach to objective image performance assessment-to historical information-theoretic methods for evaluation of imaging systems. Barrett went on to describe the many ways in which NEQ was extended to address problems beyond the simple signal-known-exactly, background-knownexactly (SEK/BKE) task.

Ken Hanson (Los Alamos National Lab.) described his years of collaboration with Bob. He said they worked together, first in the area of noise characterization of radiographic and CT images and later on the evaluation of images confounded by artifacts. In this latter work, Bob and Ken pioneered the application of a decision theoretic approach to the assessment of image reconstruction algorithms, demonstrating that the common meansquare-error metric did not predict visual task performance as measured by detectability.

Bob's contemporary work, as described by Myers, "involved the consideration of the random effects associated with multiple readers of medical images and the logical extension of this work to the problem of the evaluation of multiple competing classifiers in statistical pattern recognition. Bob tackled problems of increasing complexity over the course of his career, relying throughout on the application of a unified, decision theoretic framework. In the process he brought about consensus on the importance of a taskbased approach to the objective assessment of imaging systems."

During more than forty years of professional life, Bob Wagner made numerous contributions to the field of medical imaging that significantly impacted academia, industry, and the FDA. His brilliant mind, incredible intuition, passion for science, sense of humor, charm, and warm friendship will be greatly missed. 